\title{
ISOTOPES OF NEARLATTICES
}

\author{
Abu Saleh Abdun Noor
}

This thesis studies the nature of isotopes of a nearlattice. A nearlattice is a lower semilattice with the upper bound property, which says that any two elements possess a supremum whenever they have a common upper bound. The topic arose out of a study on the kernels, around a particular element $n$, of a skeletal congruence on a distributive lattice. Also, we found that the idea of an isotope was very fruitful in extending results on ideals of nearlattices to the $n$-ideals; that is, convex subnearlattices containing $n$.

Chapter 1 discusses ideals, join-partial congruences and other results on nearlattices which are basic to this thesis. Chapters 2 and 3 introduce the notions of standard element, neutral element and central element of a nearlattice. These concepts are essential for the further developments of Chapters 4 and 5. Also in Chapter 3, we discuss direct summands and multipliers of a nearlattice and generalize a number of results of [6] and [9].

Chapter 4 introduces the concept of an isotope. Two new types of elements arise; one is called superstandard and the other is nearly neutral. An element $n$ of a nearlattice $S$ is called medial if $m(x, n, y)=(x \wedge n) \vee(y \wedge n) \vee(x \wedge y)$ exists for all $x, y \in S$, while the nearlattice $S$ is medial if each of its elements is medial. A sesquimedial element is a strengthening of a medial element, but in a medial nearlattice every element is sesquimedial. For a medial element $n$ of a nearlattice $S$, we can form a new binary operation

Received 22 September 1980. Thesis submitted to the Flinders University of South Australia, February 1980. Degree approved September 1980. Supervisor: Dr W.H. Cornish. 
$a \cap b=m(a, n, b)$ on $S$. We show that for a medial and superstandard element $n$ of a nearlattice $S,(S ; n)$ is a semilattice. Moreover, when $n$ is nearly neutral and sesquimedial, $(S ; n)$ is in fact a nearlattice, which gives an extension of some of the results of [8] and [10]. What is more, we present converses of these results. We refer to $(S ; n)$ as an $n$-isotope, $S_{n}$, or simply an isotope of $S$.

Chapter 4 also establishes the following fundamental results about a nearlattice $S$ :

(i) for a nearly neutral and sesquimedial element $n$ of $S$, the $n$-ideals of $S$ are precisely the ideals of the isotope $S_{n}$;

(ii) when $n$ is neutral and sesquimedial, the join-partial congruences of $S$ are precisely the join-partial congruences of $S_{n}$;

(iii) if $n$ is neutral and sesquimedial in $S$, then any neutral and sesquimedial element $m$ has the same properties in $S_{n}$. Furthermore, the double isotope $\left(s_{n}\right)_{m}$ is precisely $S_{m}$.

Chapter 5 gives a characterization of the isotope $S_{n}$, when $n$ is neutral and an upper element, in the sense that $x \vee n$ exists in $S$ for all $x \in S$. Of course, every central element is upper. We show that, when $n$ is a standard element of a lattice $L$, the associated isotope is a lattice if and only if $n$ is central. This chapter also gives a nice generalization of a well known result of [3], namely, if a lattice $L$ is boolean then the isotope $L_{n}$ is also boolean and they are isomorphic. The generalization is concerned with a lattice with an involution. Chapter 6 is mainly concerned with the application of isotopes. Here, we extend a number of results on ideals of a nearlattice to its n-ideals. We discuss skeletal congruences of a distributive nearlattice and then extend the results of [1] to $n$-ideals. Chapter 7 contains the results of [2], which clarifies [7] on the ternary operation $k(a, b, c)=(a \vee(b \wedge c)) \wedge(b \vee c)$ on a modular lattice. 


\section{References}

[1] William H. Cornish, "The kernels of skeletal congruences on a distributive lattice", Math. Nachr. 84 (1978), 219-228.

[2] William H. Cornish and A.S.A. Noor, "Bands and modular lattices", Math. Sem. Notes Kobe Univ. 7 (1979), 309-326.

[3] Abraham Goetz, "On various Boolean structures in a given Boolean algebra", Publ. Math. Debrecen 18 (1971), 103-107.

[4] J. Jakubik, "Pairs of lattices with common congruence relations", Lattice theory, 171-183 (Colloquia Mathematica Societatis János Bolyai, 14. North-Holland, Amsterdam, Oxford, New York, 1976).

[5] Я. Якубин, М. Нолибиар [J. Jakubík, М. Kolibiar], "O неноторых свойствах пар струнтур" [On some properties of a pair of lattices], Caechoslovak Math. J. 4 (1954), 1-27.

[6] M.F. Janowitz, "A note on normal ideals", J. Sci. Hiroshima Univ. Ser. A-I 30 (1966), 1-9.

[7] S.A. Kiss, "Semilattices and a ternary operation in modular lattices", Bulz. Amer. Math. Soe. 54 (1948), 1176-1179.

[8] Милан Нолибиар [Milan Kolibiar], "Тернарная операция в струнтурах" [A ternary operation in lattices], Czechoslovak Math. J. 6 $(1956), 318-329$.

[9] Juhani Nieminen, "The lattice of translations on a lattice", Acta Sci. Math. 39 (1977), 109-113.

[10] Marlow Sholander, "Medians, lattices, and trees", Proc. Amer. Math. Soc. $5(1954), 808-812$. 